\title{
Fenologia e modos de polinização e dispersão de Fabaceae em floresta ciliar, Chapada Diamantina, Nordeste do Brasil
}

\section{Isys Mascarenhas Souza* \& Ligia Silveira Funch ${ }^{\mathrm{a}}$}

Programa de Pós-Graduação em Botânica, Departamento de Ciências Biológicas, Universidade Estadual de Feira de Santana, Bahia, Brasil.

\begin{abstract}
Resumo - Padrões fenológicos vêm sendo descritos com base na frequência, época e duração dos eventos, e analisados em função de fatores bióticos e abióticos, com os ciclos vegetativos e reprodutivos de espécies vegetais comumente relacionados à precipitação e ao comprimento do dia. Neste trabalho, descrevemos as estratégias fenológicas de 11 espécies de Fabaceae ocorrentes na mata ciliar do rio Lençóis, Chapada Diamantina, estado da Bahia, Brasil, e investigamos a influência dos fatores ambientais sobre os padrões fenológicos e a relação destes últimos com os modos de polinização e dispersão. A fenologia vegetativa (brotamento e queda foliar) e reprodutiva (botão, flor, fruto imaturo e fruto maduro) em 75 indivíduos foi estimada utilizando o índice de intensidade de Fournier (24 meses). A sincronia interespecífica foi calculada com base na presença ou ausência da fenofase nos indivíduos. Análises de regressão múltipla foram conduzidas para investigar a influência das variáveis ambientais sobre as fenofases, e a correlação de Pearson para verificar relações entre os eventos vegetativos e reprodutivos. As síndromes de polinização e dispersão foram definidas com base em observações de campo e na literatura. As espécies apresentaram eventos vegetativos e reprodutivos assazonais, diferindo do esperado para a família. Entretanto, as fases de flor e fruto maduro (i.e., os recursos para polinizadores e dispersores) foram sazonais. A relação com a precipitação foi positiva para o brotamento, mas negativa para a queda foliar e a dispersão dos diásporos. O brotamento, a floração e a maturação dos frutos mostraram relação positiva com o comprimento do dia. Este estudo revela a ocorrência de grupos de Fabaceae em mata ciliar com picos distintos de floração e frutificação relacionados às síndromes de polinização e dispersão, o que minimiza a sobreposição de nichos entre elas.

Palavras-chave adicionais: falenofilia, frutificação, Leguminosae, melitofilia, sazonalidade.
\end{abstract}

\begin{abstract}
Phenology and pollination and dispersal modes of Fabaceae in a riparian forest, Chapada Diamantina, Northeastern Brazil) Phenological patterns have been described based on frequency, period and duration of the events and analyzed as a function of biotic and abiotic factors with the vegetative and reproductive cycles of plant species commonly related to the precipitation and day length. In this work, we describe the phenological strategies of 11 Fabaceae species present in gallery forests along the Lençóis River, Chapada Diamantina, Bahia State, Brazil, and investigate the environmental factors influence and pollination and dispersal modes on the phenological patterns. Observations of vegetative (leaf flushing and leaf fall) and reproductive (bud, flower, immature fruit and mature fruit) phenology were made among a group of 75 individuals based on Fournier index (24 months). Interspecific synchrony was determined based on the presence or absence of each phenophase in the individuals. Multiple linear regression analyses were conducted to investigate the influence of environmental variables on the phenophases, and Pearson correlation analysis between vegetative and reproductive events. Pollination and dispersal syndromes were defined based on field observations and literature. The species demonstrated non-seasonal vegetative and reproductive events, different of the expected for the family. However, the flowers and mature fruits (i.e., resources for pollinators and dispersal agents) were seasonal. The correlation with precipitation was positive to the leaf flush and negative to leaf fall and diaspore propagation. The leaf flushing, flowering and fruit maturation are correlated to day length. Our study reveals the occurrence of different groups of Fabaceae with distinct flowering and fruiting peaks related to pollination and dispersal syndromes, which minimize the niche overlapping among them.
\end{abstract}

Additional key words: phalenophily, fruiting, Leguminosae, melittophily, seasonality.

Padrões fenológicos com base na frequência, época e duração dos eventos são comumente analisados em função de fatores abióticos (causas proximais) e bióticos (causas evolutivas), geralmente relacionados aos processos de polinização e dispersão de sementes (Newstrom et al. 1994; Williams-Linera \& Meave 2002). Vários estudos (Morellato et al. 2000; Funch et al. 2002; Borchert et al. 2004; Rivera \& Cozza 2008; Zalamea \& Gonzáles 2008; Calle et al. 2010; Miranda et al. 2011) enfatizam os fatores abióticos,

\footnotetext{
*Autora para correspondência: isys.souza@gmail.com

aligiafunch@yahoo.com

Editor responsável: Abel Augusto Conceição

Submetido: 10 jun. 2014; aceito: 21 maio 2015

Publicação eletrônica: 6 jun. 2015; versão final: 8 jun. 2015
}

evidenciando a influência dos ritmos de precipitação (associados à disponibilidade de água para as plantas) e do comprimento do dia (variável previsível) nos ciclos foliares e reprodutivos de plantas tropicais. Outros estudos (Ramírez 2002; Cerqueira et al. 2008; Stevenson et al. 2008; Fonseca et al. 2012; Souza et al. 2012) dão maior importância à polinização e dispersão na evolução dos padrões de floração e frutificação no que se refere à sazonalidade e sincronia dos eventos. Comparativamente, menor atenção tem sido dada à relação das fases foliares e reprodutivas com a época e sazonalidade dos eventos (Milla et al. 2010).

A análise fenológica é altamente dependente da escala (e.g., comunidade, população e espécie) (Newstrom et al. 1994) e dos grupos taxonômicos abordados. Famílias de plantas têm sido avaliadas 
quanto aos padrões fenológicos associados a fatores ambientais (e.g., San Martin-Gajardo \& Morellato 2003; Staggemeier et al. 2010; Bauer et al. 2012; Liuth et al. 2013); dentre elas Fabaceae (Bulhão \& Figueiredo 2002; Dutra et al. 2009), que é uma das mais importantes da flora brasileira e geralmente apresenta eventos foliares e reprodutivos sazonais, especialmente a queda foliar e maturação dos frutos no período seco (Umaña \& Alencar 1993; Bulhão \& Figueiredo 2002; Pedroni et al. 2002; Locatelli \& Machado 2004; Cruz Neto et al. 2007; Costa \& Morais 2008; Oliveira \& Sigrist 2008; Pinto et al. 2008; Borges et al. 2009; Dutra et al. 2009; Guedes et al. 2009; Navarro et al. 2009; Souza et al. 2012). Entretanto, considerando a riqueza e abundância de Fabaceae nos diferentes ecossistemas brasileiros (Lima et al. 2014), as abordagens fenológicas realizadas até o momento configuram ainda um painel pouco representativo para a compreensão das estratégias foliares e reprodutivas na família.

Neste estudo, nós investigamos os padrões fenológicos de 11 espécies arbustivo-arbóreas de Fabaceae encontradas em floresta ciliar na Chapada Diamantina, Bahia, considerando suas relações com fatores ambientais e os modos de polinização e dispersão. Nossas principais questões foram: 1- Os padrões vegetativos e reprodutivos são sazonais? 2- A fenologia das espécies está relacionada às variáveis ambientais precipitação, temperatura e comprimento do dia? 3- Os caracteres eco-morfológicos relacionados aos modos de polinização e dispersão contribuem para a modulação dos padrões fenológicos? 4- A relação entre as fenofases vegetativa e reprodutiva configura uma estratégia fenológica para o grupo?

\section{MATERIAL E MÉtodos}

Área de estudo. O trecho de floresta ciliar do rio Lençóis estudado na Chapada Diamantina, Bahia, Brasil

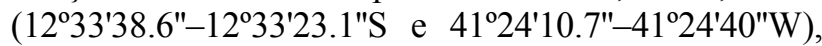
ocorre em solo distrófico litólico, a cerca de $500 \mathrm{~m}$ s.n.m., correspondendo a uma faixa estreita de 15-25 m de vegetação paralela à calha do rio, com dossel alcançando aproximadamente $20 \mathrm{~m}$ de altura e subbosque com espécies arbustivas e arbóreas variando de 3,5-8,0 $\mathrm{m}$ de altura, composto principalmente por espécies perenifólias (Funch et al. 2002). Vinte espécies lenhosas de árvores, arbustos e trepadeiras de Fabaceae foram inventariadas nessa floresta (Funch \& Oliveira 2011), sendo a maioria delas encontrada em outras florestas na Chapada Diamantina (Funch et al. 2008; Ribeiro-Filho et al. 2009; Couto et al. 2011).

A floresta estudada encontra-se no domínio da Caatinga (Juncá et al. 2005) e o clima da região é tropical do tipo Aw (Alvares et al. 2013), com pequena variação de temperatura média $\left(22-25^{\circ} \mathrm{C}\right)$ ao longo do ano, predomínio de chuvas no verão (novembro a março) e seca bem marcada no inverno (junho a outubro), evidenciando uma sazonalidade climática (Funch et al. 2002). Além disso, a região apresenta dias mais curtos no inverno (maio a julho) e dias mais longos no verão (novembro a janeiro). Os dados de temperatura e precipitação mensais da estação de Lençóis (Figura 1), a cerca de $1 \mathrm{~km}$ da área de estudo, foram fornecidos pelo Instituto Nacional de Meteorologia (INMET). As médias de comprimento do dia foram extraídas do USNO (Astronomical Applications Department), com base nos dados de latitude e longitude da área de estudo.

Fenologia. As observações foram realizadas mensalmente durante 24 meses (nov. 2008 - out. 2010), em 75 indivíduos adultos com diâmetro à altura do peito maior ou igual a $3 \mathrm{~cm}$, situados ao longo de trilhas paralelas à calha do rio, pertencentes a 11 espécies de Fabaceae, a segunda família mais representativa em número de espécies na área de estudo (Funch, dados não publicados). As espécies selecionadas para o acompanhamento fenológico representam $85 \%$ das espécies do componente arbustivo-arbóreo e 55\% do componente lenhoso da floresta. $\mathrm{O}$ número de indivíduos por espécie monitorada variou conforme a abundância e distribuição das espécies na área. Amostras das espécies foram coletadas e depositadas na coleção do Herbário da Universidade Estadual de Feira de Santana

A

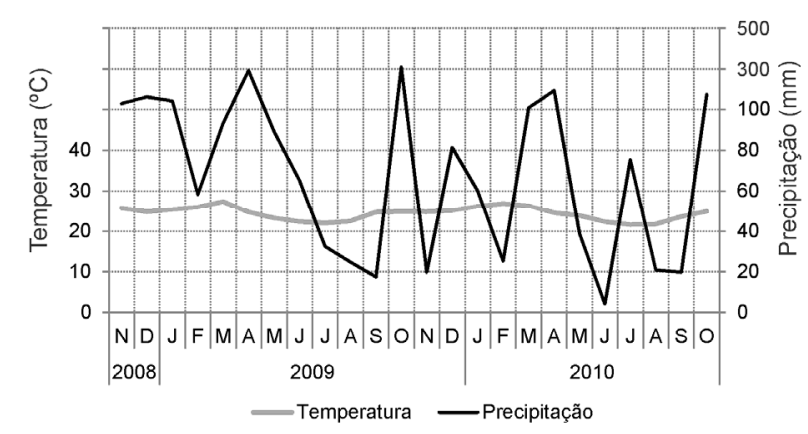

$\mathrm{B}$

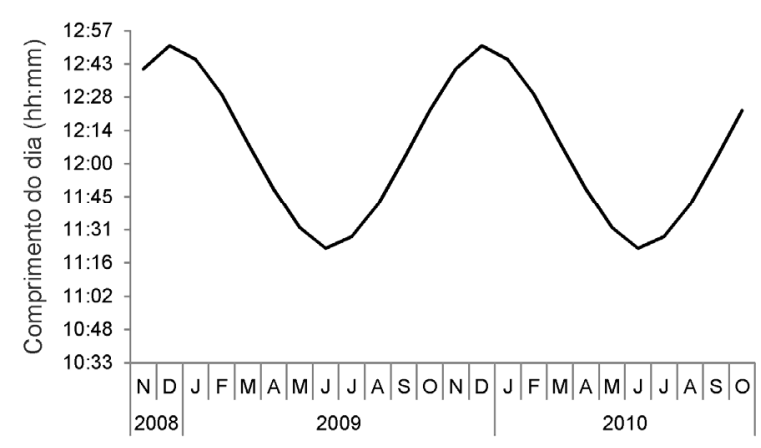

Figura 1. Variáveis ambientais em Lençóis, Chapada Diamantina, Bahia, Brasil (nov. 2008 a out. 2010): A- climatograma higrotérmico (fonte dos dados de precipitação total e temperatura média mensal: INMET); B- médias mensais de comprimento do dia (USNO - Astronomical Applications Department, disponível em http://aa.usno.navy.mil/data/docs/RS_OneYear.php). 
(HUEFS). Foram acompanhadas fenofases vegetativas (queda foliar e brotamento) e reprodutivas (botão, flor, fruto imaturo e fruto maduro). A intensidade de cada fenofase foi estimada de acordo com o índice de Fournier (1974). Os padrões fenológicos foliares foram categorizados como decíduo, semidecíduo e perenifólio episódico (Frankie et al. 1974), e os reprodutivos como contínuo, anual, subanual e supra-anual (Newstrom et al. 1994). A sincronia interespecífica dos eventos foi calculada pelo "índice de atividade" (ou porcentagem de indivíduos) (Bencke \& Morellato 2002).

Síndromes de polinização e dispersão. As síndromes de polinização foram identificadas com base na forma, simetria, coloração e odor das flores (Faegri \& van der Pijl 1979). Já que as síndromes florais definidas apenas com base nas características da flor nem sempre refletem os reais polinizadores (Tripp \& Manos 2008; Obermuller et al. 2008; Ollerton et al. 2009), também foram utilizadas observações de campo e informações na literatura sobre a polinização dessas espécies ou de espécies próximas. As síndromes de dispersão basearam-se na morfologia dos diásporos (van der Pijl 1982), considerando especialmente características do pericarpo (coloração, consistência e deiscência) e da semente (acessórios da testa e presença de ala). No caso das espécies que não floresceram e/ou frutificaram, as síndromes foram definidas apenas com base na literatura.

Análise dos dados. A possível influência das variáveis ambientais (precipitação total, médias mensais de temperatura e comprimento do dia) sobre cada fenofase foi avaliada por análises de regressão múltipla. Nestas análises, a variável temperatura foi excluída por apresentar correlação com o comprimento do dia $(r=0,78 ; p<0,05)$. A relação entre as fenofases vegetativas e reprodutivas foi testada pela correlação de Pearson. Foi utilizado o programa R, versão 3.1.0 para Windows ( $\mathrm{R}$ Core Team 2014) para os testes de normalidade e heterogeneidade dos dados, bem como análises de regressão múltipla e correlação. Os dados que apresentaram distribuição heterogênea dos resíduos foram transformados para logaritmo a fim de garantir uma maior homogeneização e a confiabilidade dos resultados nos testes de regressão subsequentes.

A ocorrência e intensidade da sazonalidade de cada fenofase foram avaliadas para os dois anos de estudo por meio de análises estatísticas circulares utilizando o programa ORIANA 4.02, versão demonstração (Kovach Computing Services; disponível em http://www.kovcomp.co.uk). Neste procedimento, os meses foram convertidos em ângulos com intervalos de $30^{\circ}$ para cada observação. A frequência de ocorrência dos indivíduos foi computada dentro de cada mês e, em seguida, foram calculados o ângulo médio $(u)$ (posteriormente convertido em data média), o comprimento do vetor médio $(r)$, o desvio padrão circular e a significância do ângulo médio para cada ano estudado (teste de Rayleigh $z$ ) (Morellato et al. 2010). O ângulo médio representa a data média da atividade dos eventos fenológicos. O vetor $r$ representa o grau de agregação temporal dos dados fenológicos, de modo que são consideradas agregadas ou sazonais as fenofases com valores superiores a 0,5 para esse parâmetro. O teste de Rayleigh $(z)$ é utilizado para determinar se os ângulos ou datas estão distribuídos uniformemente ao longo do ano, pois se os dados estiverem distribuídos de forma contínua, não há sazonalidade (Morellato et al. 2010).

\section{RESULTADOS}

Fenologia vegetativa. Foram identificados três padrões de fenologia foliar para as espécies de Fabaceae estudadas na floresta ciliar do rio Lençóis: 1perenifólio episódico (72,7\% das espécies); 2- decíduo $(18,2 \%)$; e 3- semidecíduo $(9,1 \%)$ (Tabela 1). A família apresentou padrão perenifólio episódico, assazonal para os eventos foliares (Tabela 2). Os episódios de queda foliar foram mais concentrados nos períodos secos e o brotamento coincidiu com as máximas de precipitação, em baixa intensidade durante todo o período de estudo (Figura 2). Queda foliar e brotamento apresentaram relação com a precipitação (Tabela 3), com alta sincronia interespecífica nos meses mais secos (Figura 2).

Fenologia reprodutiva. As espécies de Fabaceae apresentaram diferentes padrões de floração e frutificação (Tabela 1): floração com frequência anual (45,5\%), supra-anual $(27,3 \%)$, subanual $(18,2 \%)$ e contínua $(9 \%)$, e duração breve $(45,4 \%)$, intermediária $(36,4 \%)$ e longa $(9,1 \%)$; e frutificação com frequência anual (54,5\%), supra-anual $(27,3 \%)$ e subanual $(18,2 \%)$, e duração longa $(45,5 \%)$, intermediária $(27,3 \%)$ e breve $(18,2 \%)$. A família exibiu padrão de floração e frutificação contínuo, com baixa sincronia interespecífica durante o período de estudo (Figura 2). A oferta de botões e frutos imaturos foi contínua, mas a disponibilidade de flores e frutos maduros foi anual, sazonal (Tabela 2), com duração intermediária à longa (Figura 2). Os picos de floração (botões e flores) e dispersão dos diásporos coincidiram com os meses de maior e menor precipitação, respectivamente (Figura 2). A maturação dos frutos mostrou relação com a precipitação e o brotamento, a floração (botões e flores) e os frutos maduros apresentaram relação com comprimento do dia (Tabela 3 ).

Relações entre os eventos foliares e reprodutivos. Nas Fabaceae estudadas, foram verificadas as seguintes correlações entre as fenofases foliares e reprodutivas: 1- brotamento botão $(r=0,60 ; p<0,05)$; 2brotamento flor $(r=0,51 ; p<0,05)$; e 3- queda foliar frutos maduros $(r=0,63 ; p<0,05)$. 


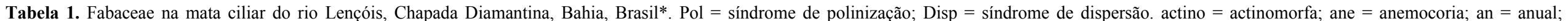

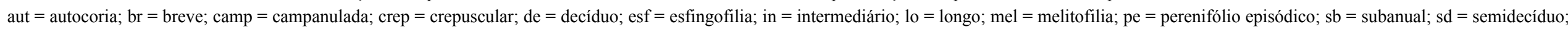
$\mathrm{sp}$ = supra-anual; zigom = zigomorfa; zoo = zoocoria. Tamanho da flor (distância da base do receptáculo ao ápice da corola): pequeno $<14 \mathrm{~mm}$; grande $\geq 14 \mathrm{~mm}$.

\begin{tabular}{|c|c|c|c|c|c|c|c|c|c|c|c|c|c|}
\hline & \multirow{2}{*}{ Hábito } & \multirow{2}{*}{$\mathbf{N}$} & \multicolumn{3}{|c|}{ Fenologia } & \multicolumn{4}{|c|}{ Caracteres florais } & \multirow{2}{*}{$\begin{array}{l}\text { Antese } \\
\text { (início) }\end{array}$} & \multirow{2}{*}{ Pol } & \multirow{2}{*}{ Disp } & \multirow{2}{*}{$\begin{array}{l}\text { Referência para as } \\
\text { síndromes de polinização }\end{array}$} \\
\hline & & & Foliar & Flor & Fruto & Tamanho & Forma & Simetria & Cor & & & & \\
\hline $\begin{array}{l}\text { Anadenanthera colubrina (Vell.) } \\
\text { Brenan }\end{array}$ & Arbóreo & 10 & de & $\mathrm{sp}, \mathrm{br}$ & sp, lo & pequeno & camp & actino & creme & - & mel & ane & Quirino \& Machado (2014) \\
\hline $\begin{array}{l}\text { Balizia pedicellaris (DC.) Barneby } \\
\& \text { Grimes }\end{array}$ & Arbóreo & 11 & pe & an, in & an, lo & pequeno & camp & actino & creme & - & esf & aut & Dick et al. (2008) \\
\hline Calliandra hirtiflora Benth. & Arbustivo & 4 & pe & an, lo & an, in & pequeno & camp & actino & creme & crep & esf & aut & $\begin{array}{l}\text { Cruden et al. (1976); } \\
\text { Matthews \& Hopkinson }\end{array}$ \\
\hline Calliandra lintea Berneby & Arbustivo & 5 & pe & co & $\mathrm{sb}, \mathrm{br}$ & pequeno & camp & actino & creme & crep & esf & aut & (1998); Hernandez-Conrique \\
\hline Calliandra parvifolia Benth. & Arbustivo & 7 & pe & $\mathrm{sb}$, in & $\mathrm{sb}$, in & pequeno & camp & actino & creme & crep & esf & aut & et al. (2007) \\
\hline Inga cayennensis Sagot ex Benth. & Arbóreo & 12 & pe & an, in & an, in & pequeno & camp & actino & creme & crep & esf & zoo & $\begin{array}{l}\text { Koptur (1984); Barros et al. } \\
\text { (2013) }\end{array}$ \\
\hline Plathymenia reticulata Benth. & Arbóreo & 2 & sd & an, br & an, lo & pequeno & camp & actino & creme & - & mel & ane & Goulart et al. (2005) \\
\hline Andira fraxinifolia Benth. & Arbóreo & 2 & pe & $\mathrm{sb}, \mathrm{br}$ & an, in & grande & plana & zigom & lilás & diurna & mel & zoo & Frankie et al. (1976) \\
\hline Bowdichia virgilioides Kunth & Arbóreo & 7 & de & $\mathrm{sp}, \mathrm{br}$ & $\mathrm{sp}, \mathrm{br}$ & grande & plana & zigom & roxo & diurna & mel & ane & Silva et al. (2011) \\
\hline $\begin{array}{l}\text { Hymenolobium janeirense Kuhl. } \\
\text { var. stipulatum (N.Mattos) Lima }\end{array}$ & Arbóreo & 5 & pe & $\mathrm{sp}, \mathrm{br}$ & sp, - & grande & plana & zigom & rosa & diurna & mel & ane & $\begin{array}{l}\text { Funch et al. (dados não } \\
\text { publicados) }\end{array}$ \\
\hline $\begin{array}{l}\text { Senna cana (Nees \& Mart.) } \\
\text { H.S.Irwin \& Barneby }\end{array}$ & Arbustivo & 10 & pe & an, in & an, lo & grande & plana & zigom & amarelo & diurna & mel & aut & Souza et al. (2012) \\
\hline
\end{tabular}


Tabela 2. Sazonalidade em Fabaceae na mata ciliar do rio Lençóis, Chapada Diamantina, Bahia, Brasil (nov. 2008 a out. 2010 ). Qf = queda foliar; $\mathrm{Br}=$ brotamento; $\mathrm{Bo}=$ botão; $\mathrm{Fl}=$ flor; $\mathrm{Fi}=$ fruto imaturo; Fm = fruto maduro; Fal = falenofilia; Mel = melitofilia; Anem $=$ anemocoria; Auto $=$ autocoria; Zooc $=$ zoocoria; $(* * * * *)=$ valor inexistente devido ao baixo número de observações.

\begin{tabular}{|c|c|c|c|c|c|c|c|c|c|c|c|c|}
\hline Ano & Parâmetros estatísticos & Qf & $\mathrm{Br}$ & Bo & Fl & $\mathbf{F i}$ & $\mathbf{F m}$ & Fal & Mel & Anem & Auto & Zooc \\
\hline \multirow[t]{8}{*}{$1^{\circ}$} & Número de observações & 275 & 327 & 102 & 50 & 107 & 134 & 154 & 2 & 20 & 75 & 26 \\
\hline & Vetor médio $(\mu)$ & $242,964^{\circ}$ & $350,884^{\circ}$ & $303,598^{\circ}$ & $307,445^{\circ}$ & $110,429^{\circ}$ & $185,459^{\circ}$ & $306,966^{\circ}$ & $135^{\circ}$ & $343,898^{\circ}$ & $204,482^{\circ}$ & $115,622^{\circ}$ \\
\hline & Grupo médio & Set. & Dez. & Nov. & Nov. & Abr. & Jul. & Nov. & Maio & Dez. & Jul. & Abr. \\
\hline & Data média & $2 /$ set. & $21 /$ dez. & $3 /$ nov. & 7/nov. & 21/abr. & 6/jul. & 6/nov. & $16 /$ maio & 13/dez. & $25 /$ jul. & 26/abr. \\
\hline & Compr. do vetor médio $(r)$ & 0,339 & 0,107 & 0,354 & 0,743 & 0,366 & 0,292 & 0,512 & 1 & 0,705 & 0,578 & 0,528 \\
\hline & Concentração & 0,72 & 0,216 & 0,758 & 2,308 & 0,786 & 0,61 & 1,187 & $* * * * *$ & 2,035 & 1,423 & 1,236 \\
\hline & Desvio padrão circular & $84,321^{\circ}$ & $121,014^{\circ}$ & $82,544^{\circ}$ & $44,142^{\circ}$ & $81,263^{\circ}$ & $89,958^{\circ}$ & $66,308^{\circ}$ & $* * * * *$ & $47,95^{\circ}$ & $59,975^{\circ}$ & $64,789^{\circ}$ \\
\hline & Teste de Rayleigh $(p)$ & 0,000 & 0,023 & 0,000 & 0,000 & 0,000 & 0,000 & 0,000 & 0,137 & 0,000 & 0,000 & 0,000 \\
\hline \multirow[t]{8}{*}{$2^{\circ}$} & Número de observações & 487 & 177 & 130 & 54 & 75 & 115 & 144 & 40 & 15 & 79 & 16 \\
\hline & Vetor médio $(\mu)$ & $222,666^{\circ}$ & $354,367^{\circ}$ & $332,389^{\circ}$ & $11,145^{\circ}$ & $107,633^{\circ}$ & $198,326^{\circ}$ & $329,036^{\circ}$ & $33,912^{\circ}$ & $262,737^{\circ}$ & $204,837^{\circ}$ & $110,622^{\circ}$ \\
\hline & Grupo médio & Ago. & Dez. & Dez. & Jan. & Abr. & Jul. & Nov. & Fev. & Set. & Jul. & Abr. \\
\hline & Data média & 13/ago. & $25 /$ dez. & $2 /$ dez. & $11 /$ jan. & 18/abr. & 19/jul. & 29/nov. & $3 /$ fev. & $22 /$ set. & 26/jul. & $21 /$ abr. \\
\hline & Compr. do vetor médio $(r)$ & 0,138 & 0,351 & 0,338 & 0,306 & 0,304 & 0,425 & 0,345 & 0,377 & 0,818 & 0,462 & 0,622 \\
\hline & Concentração & 0,279 & 0,751 & 0,719 & 0,643 & 0,639 & 0,939 & 0,736 & 0,814 & 2,514 & 1,039 & 1,603 \\
\hline & Desvio padrão circular & $114,026^{\circ}$ & $82,86^{\circ}$ & $84,366^{\circ}$ & $88,186^{\circ}$ & $88,403^{\circ}$ & $74,943^{\circ}$ & $83,536^{\circ}$ & $80,046^{\circ}$ & $36,27^{\circ}$ & $71,237^{\circ}$ & $55,817^{\circ}$ \\
\hline & Teste de Rayleigh $(p)$ & 0,000 & 0,000 & 0,000 & 0,006 & 0,000 & 0,000 & 0,000 & 0,003 & 0,000 & 0,000 & 0,001 \\
\hline
\end{tabular}

Síndromes de polinização e fenologia da floração. A melitofilia foi identificada em 54,5\% das espécies, sendo bem representada entre as Papilionoideae e "Caesalpinioideae". Entre as Mimosoideae, a falenofilia predominou em 45,5\% das espécies (Tabela 1). A floração foi sazonal em ambas as síndromes apenas no primeiro ano de estudo (Tabela 2), sendo nas melitófilas mais expressiva no segundo ano, coincidindo com os maiores valores de comprimento do dia (Figura 2). Já as espécies falenófilas floresceram ao longo dos dois anos estudados, com picos de floração concomitantes com os picos de brotamento e o aumento do comprimento do dia, pouco antes da floração das melitófilas (Figura 2).

Síndromes de dispersão e fenologia da frutificação. As espécies de Fabaceae foram classificadas em autocóricas (45,4\%), anemocóricas $(36,4 \%)$ e zoocóricas (18,2\%) (Tabela 1). Foi verificado um padrão sazonal de frutificação para os grupos estabelecidos com base nas síndromes de dispersão (Tabela 2). Contudo, esses grupos divergiram quanto ao período de frutificação (grupo médio e data média) (Tabela 2). As espécies com dispersão abiótica (autocóricas e anemocóricas) liberaram os diásporos nos períodos com menor disponibilidade hídrica, enquanto as espécies com dispersão biótica liberaram nos períodos mais chuvosos (Figura 2).

\section{DISCUSSÃo}

O padrão perenifólio episódico identificado na maioria das espécies de Fabaceae acompanhadas no presente estudo difere da sazonalidade foliar marcada que vem sendo descrita para a família (Umaña \& Alencar 1993; Bulhão \& Figueiredo 2002; Pedroni et al. 2002; Locatelli \& Machado 2004). Este padrão pode ser interpretado como reflexo à condição ciliar a qual essas espécies estão sujeitas. Mesmo nos períodos mais secos, a pouca disponibilidade hídrica nas matas ciliares não se compara à escassez hídrica nos ambientes marcadamente sazonais em relação à precipitação e disponibilidade de água. Esse mesmo padrão perenifólio, com produção e perda descontínuas (episódicas) de folhas e tendência à concentração em determinadas épocas do ano, também se aplica a espécies de outras famílias do componente arbustivoarbóreo da floresta ciliar do rio Lençóis (Funch et al. 2002), o que ressalta a relação entre a fenologia vegetativa e a presença de curso d'água.

A influência de fatores como precipitação, temperatura e comprimento do dia sobre os padrões fenológicos foliares em árvores tropicais já é bem conhecida (Janzen et al. 1967; Frankie et al. 1974; Talora \& Morellato 2000). Entretanto, em florestas ciliares tropicais caracterizadas pelo predomínio de espécies perenifólias, a deciduidade não é tão evidente (Frankie et al. 1974). Em regiões sazonais como neste estudo, a perda episódica de folhas por espécies perenifólias é intensificada com o aumento da estiagem provocada pela diminuição da precipitação e o aumento nos déficits de pressão de vapor durante a estação seca (Reich 1995). Esta relação entre atividades foliares e precipitação ficou evidente aqui, já que as análises estatísticas apontaram uma relação significativa entre elas. Além da precipitação, o comprimento do dia também apresentou relação com o brotamento. Como o comprimento do dia está 


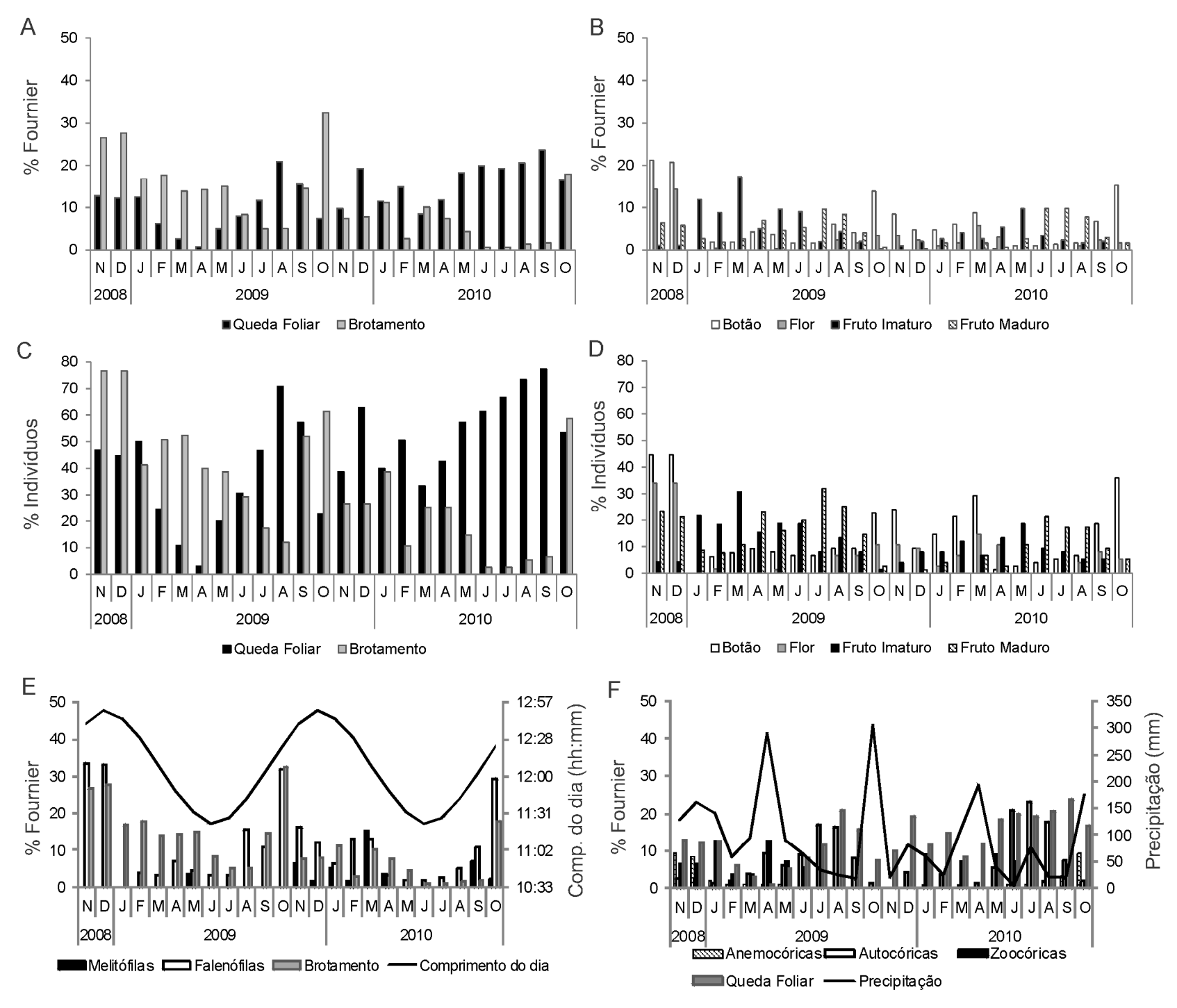

Figura 2. Fenologia das espécies de Fabaceae na mata ciliar do rio Lençóis, Chapada Diamantina, Bahia, Brasil (nov. 2008 a out. 2010): A- intensidade das fenofases vegetativas. B- intensidade das fenofases reprodutivas; C- sincronia interespecífica das fenofases vegetativas; D- sincronia interespecífica das fenofases reprodutivas; E- síndromes de polinização; F- síndromes de dispersão.

Tabela 3. Análise de regressão múltipla entre as fenofases observadas em Fabaceae e variáveis ambientais da região de Lençóis (resultados significativos em negrito), Chapada Diamantina, Bahia, Brasil (nov. 2008 a out. 2010).

\begin{tabular}{lllllll}
\hline & \multicolumn{3}{c}{ Precipitação } & \multicolumn{2}{c}{ Fotoperíodo } & $\mathbf{R}^{\mathbf{2}} \mathbf{( \% )}$ \\
\hline Queda foliar & $\mathbf{F}=\mathbf{1 2 , 6 8 4 0}$ & $\mathbf{p}=\mathbf{0 , 0 0 1 4 5 1}$ & $\mathrm{F}=0,4209$ & $\mathbf{p}=0,522170$ & 33,51 \\
Brotamento & $\mathbf{F}=\mathbf{1 2 , 9 8 6 8}$ & $\mathbf{p}=\mathbf{0 , 0 0 1 3 0 2}$ & $\mathbf{F}=\mathbf{4 , 5 0 0 7}$ & $\mathbf{p}=\mathbf{0 , 0 4 3 5 7 4}$ & 40,21 \\
Botão & $\mathrm{F}=4,0300$ & $\mathrm{p}=0,055196$ & $\mathbf{F}=\mathbf{7 , 9 3 0 6}$ & $\mathbf{p}=\mathbf{0 , 0 0 9 1 5 2}$ & 31,51 \\
Antese & $\mathrm{F}=1,9947$ & $\mathrm{p}=0,16971$ & $\mathbf{F}=\mathbf{7 , 1 6 9 7}$ & $\mathbf{p}=\mathbf{0 , 0 1 2 6 7}$ & 26,06 \\
Fruto imaturo & $\mathrm{F}=0,2514$ & $\mathrm{p}=0,6203$ & $\mathrm{~F}=2,8006$ & $\mathbf{p}=0,1062$ & - \\
Fruto maduro & $\mathbf{F}=\mathbf{6 , 6 5 4 2}$ & $\mathbf{p}=\mathbf{0 , 0 1 5 9 0}$ & $\mathbf{F}=\mathbf{5 , 8 7 2 3}$ & $\mathbf{p}=\mathbf{0 , 0 2 2 6 4}$ & 32,51 \\
\hline
\end{tabular}

fortemente correlacionado à temperatura, ambos parecem influenciar o início da produção de folhas nas espécies estudadas.

Os eventos reprodutivos de Fabaceae foram contínuos durante todo o período de estudo, diferentemente do que vem sendo descrito para as espécies da família, as quais costumam apresentar comportamento sazonal (Bulhão \& Figueiredo 2002; Funch et al. 2002; Oliveira \& Sigrist 2008; Dutra et al. 2009). Entretanto, quando considerados os modos de polinização e dispersão, observou-se que a oferta de recursos (flores e frutos maduros) em cada síndrome 
foi mais concentrada em períodos distintos e subsequentes do ano, configurando um padrão sazonal para a disponibilidade de flores (no primeiro ano) e dispersão dos diásporos nas diferentes síndromes.

Diferentes estratégias de polinização estão relacionadas a distintos padrões de floração (Gentry 1974). As espécies melitófilas e falenófilas de Fabaceae estudadas aqui, embora sazonais, floresceram em épocas distintas e exibiram diferentes padrões fenológicos. As falenófilas disponibilizaram flores ao longo do ano, com períodos de floração mais intensa, enquanto as melitófilas tiveram floração concentrada em determinado período, sazonalidade essa que parece ser importante para a manutenção da fauna de abelhas (Dutra et al. 2009). Os distintos picos de floração relacionados às síndromes de polinização detectados aqui sugerem uma segregação temporal da fauna de polinizadores associada a cada grupo de espécies em Fabaceae, o que minimiza a sobreposição entre seus nichos.

Alguns autores constataram uma associação entre abelhas pequenas e flores inconspícuas e com baixa produção de néctar (Primack 1987; Viana \& Kleinert 2006). As espécies melitófilas de Mimosoideae analisadas neste estudo apresentaram esta associação. As demais espécies melitófilas, com flores relativamente vistosas, grandes e zigomorfas, estão associadas a abelhas de grande porte, como proposto por Frankie et al. (1983) e confirmado em vários outros estudos. Por exemplo, Senna cana (Nees \& Mart.) H.S.Irwin \& Barneby, uma das espécies melitófilas com flores grandes deste estudo, apresenta uma estreita associação com abelhas de grande porte do gênero Xylocopa (Souza et al. 2012).

A relação significativa entre maturação dos frutos e baixa precipitação obtida para Fabaceae neste estudo provavelmente reflete a predominância de espécies anemocóricas e autocóricas na família. Essa relação já é bem conhecida para as espécies de Fabaceae com diásporos dispersos por agentes abióticos (Bulhão \& Figueiredo 2002). A maturação dos frutos secos ocorre em conformidade com a queda nas taxas de precipitação e umidade relativa do ar (Janzen 1967; Croat 1969; Morellato \& Leitão-Filho 1990), favorecendo a dessecação dos frutos e a subsequente dispersão dos diásporos (Janzen 1967).

A relação entre as fases foliares e reprodutivas nas Fabaceae investigadas aqui foi evidente, com correlações positivas entre brotamento e floração e entre queda foliar e maturação dos frutos. Esta última relação ("deciduidade dispersão") é comum em espécies com frutos secos (anemocóricos e autocóricos) e aumenta a eficiência da dispersão, uma vez que a redução das copas amplia a exposição dos diásporos ao vento (Marques et al. 2004; Leyser et al. 2009). Já na relação entre brotamento foliar e floração, os picos de brotamento que antecedem brevemente o início da floração resultam do desenvolvimento das gemas terminais em inflorescências, promovendo assim a necessária alternância entre as fases foliar e reprodutiva. Tanto o brotamento quanto a floração tiveram relação significativa positiva com o comprimento do dia, o qual já tem um papel reconhecido como "gatilho" para o desencadeamento desses eventos fenológicos em muitas espécies (Morellato et al. 2000; Talora \& Morellato 2000; Pedroni et al. 2002; Pereira et al. 2008).

Nossos resultados mostram que a condição ciliar das espécies de Fabaceae analisadas neste estudo contribui para a existência de padrões foliares e reprodutivos assazonais, contrastando com os padrões sazonais usualmente descritos para a família. Ainda assim, a relação dos eventos fenológicos dessas espécies com a precipitação, o comprimento do dia e a temperatura são evidentes. As fases flor e fruto maduro quando analisadas isoladamente, de modo a refletir os modos de polinização e dispersão, apresentam padrões sazonais. Grupos associados aos diferentes modos de polinização com picos distintos de floração parecem minimizar a sobreposição entre o nicho dessas espécies e reforça o papel dos polinizadores na modulação desse evento. Finalmente, a frutificação associada à queda foliar favorece a dispersão dos diásporos, que são predominantemente autocóricos e anemocóricos.

\section{AgradeCIMENTOS}

À Universidade Estadual de Feira de Santana, pela infraestrutura utilizada para o desenvolvimento deste trabalho. Ao pesquisador Roy Funch pelo apoio nas estadias em Lençóis durante as atividades de campo, bem como pelo auxílio na elaboração do abstract deste artigo. Esta pesquisa teve suporte da Fundação de Amparo à Pesquisa do Estado da Bahia (FAPESB 5303/2009) e do Conselho Nacional de Desenvolvimento Científico e Tecnológico (CNPq 480508/2008). I.M.S. teve bolsa da FAPESB e L.S.F. teve bolsa (PQ2) do CNPq.

\section{REFERÊNCIAS}

Alvares, C.A.; Stape, J.L.; Centelhas, P.C.; Gonçalves, J.L.M. \& Sparover, G. 2013. Köppen's climate classification map for Brazil. Meteorologische Zeitschrift 22(6): 711-728.

Barros, E.C.O; Webber, A.C. \& Machado, I.C. 2013. Limitação de polinizadores e mecanismo de autoincompatibilidade de ação tardia como causas da baixa formação de frutos em duas espécies simpátricas de Inga. Rodriguésia 64(1): 37-47.

Bauer, D.; Goetz, M.N.B.; Müller, A. \& Schmitt, J.L. 2012. Fenologia de três espécies de Myrsine L. em floresta secundária semidecídua no Sul do Brasil. Revista Árvore 36(5): 859-868.

Bencke, C.S.C. \& Morellato, L.P.C. 2002. Comparação de dois métodos de avaliação da fenologia de plantas, sua interpretação e representação. Revista Brasileira de Botânica 25(3): 269-276.

Borchert, R.; Meyer, S.A.; Felger, R.S. \& Porter-Bolland, L. 2004. Environmental control of flowering periodicity in Costa 
Rican and Mexican tropical dry forests. Global Ecology and Biogeography 13: 409-425.

Borges, L.A.; Sobrinho, M.S. \& Lopes, A.V. 2009. Phenology, pollination, and breeding system of the threatened tree Caesalpinia echinata Lam. (Fabaceae), and a review of studies on the reproductive biology in the genus. Flora 204: 111-130.

Bulhão, C.F. \& Figueiredo, P.S. 2002. Fenologia de leguminosas arbóreas em uma área de cerrado marginal no Nordeste do Maranhão. Revista Brasileira de Botânica 25(3): 361-369.

Calle, Z.; Schlumpberger, B.O.; Piedrahita, L.; Leftin, A.; Hammer, S.A.; Tye, A. \& Borchert, R. 2010. Seasonal variation in daily insolation induces synchronous bud break and flowering in the tropics. Trees 24: 865-877.

Cerqueira, C.O.; Funch, L.S. \& Borba, E.L. 2008. Fenologia de Syngonanthus mucugensis Giul. subsp. mucugensis e $S$. curralensis Moldenke (Eriocaulaceae), nos municípios de Mucugê e Morro do Chapéu, Chapada Diamantina, BA, Brasil. Acta Botanica Brasilica 22(4): 962-969.

Costa, R.A.C.V. \& Morais, A.B.B. 2008. Fenologia e visitantes florais de Erythrina crista-galli L. (Leguminosae, Faboideae) em Santa Maria, Rio Grande do Sul, Brasil. Biotemas 21(2): $51-56$.

Couto, A.P.L.; Funch, L.S. \& Conceição, A.A. 2011. Composição florística e fisionomia de floresta estacional semidecídua submontana na Chapada Diamantina, Bahia, Brasil. Rodriguésia 61(2): 391-405.

Croat, T.B. 1969. Seasonal flowering behavior in Central Panama. Annals of Missouri Botanical Garden 56: 295-307.

Cruden, R.W.; Kinsman, S.; Stockhouse, R.E. \& Linhart, Y.B. 1976. Pollination, fecundity, and the distribution of mothflowered plants. Biotropica 8(3): 204-210.

Cruz Neto, O.; Lopes, A.V.F. \& Machado, I.C. 2007. Ecologia da polinização de Inga striata (Benth.) (Leguminosae-Mimosoideae) em um remanescente de mata atlântica no Nordeste do Brasil. Revista Brasileira de Biociências 5(1): 570-572.

Dick, C.W.; Olivier, J.H.; Jones, F.A. \& Rémy, J.P. 2008. Spatial scales of pollen and seed-mediated gene flow in Tropical Rain Forest trees. Tropical Plan Biology 1: 20-33.

Dutra, V.F.; Vieira, M.F.; Garcia, F.C.P. \& Lima, H.C. 2009. Fenologia reprodutiva, síndromes de polinização e dispersão em espécies de Leguminosae dos campos rupestres do Parque Estadual do Itacolomi, Minas Gerais, Brasil. Rodriguésia 60(2): 371-387.

Faegri, K. \& van der Pijl, L. 1979. The Principles of Pollination Ecology. Pergamon Press, Oxford.

Fonseca, R.B.R.; Funch, L.S. \& Borba, E.L. 2012. Dispersão de sementes de Melocactus glaucescens e M. paucispinus (Cactaceae), no Município de Morro do Chapéu, Chapada Diamantina-BA. Acta Botanica Brasilica 26(2): 481-492.

Fournier, L.A. 1974. Um método cuantitativo para la medición de características fenológicas em árbores. Turrialba 24: 422-423.

Frankie, G.W.; Haber, W.A.; Opler, P.A. \& Bawa, K.S. 1983. Characteristics and organization of the large bee pollination system in the Costa Rican dry forest. In: C.E. Jones \& R.J. Little (eds), Handbook of Experimental Pollination Biology. Van Nostrand Reinhold Company, Nova York, p. 411-447.

Frankie, G.W.; Baker, H.G. \& Opler, P.A. 1974. Comparative phenological studies of trees in tropical lowland wet and dry forest sites of Costa Rica. Journal of Ecology 62: 881-913.
Frankie, G.W.; Opler, P.A. \& Bawa, K.S. 1976. Foraging behaviour of solitary bees: implications for outcrossing of a neotropical forest tree species. Journal of Ecology 64(3): 10491057.

Funch, L.S. \& Oliveira, R.P. 2011. Angiospermas: plantas com flores e frutos. In: L.S. Funch \& L.A.P. Miranda (orgs), Serrano - Parque Municipal da Muritiba. Radami, Feira de Santana, p. 111-137.

Funch, L.S.; Funch, R. \& Barroso, G.M. 2002. Phenology of gallery and montane forest in the Chapada Diamantina, Bahia, Brazil. Biotropica 34(1): 40-50.

Funch, L.S.; Rodal, M.J.N. \& Funch, R.R. 2008. Floristic aspects of forests of the Chapada Diamantina, Bahia, Brazil. In: W. Thomas \& E.G. Britton (orgs), The Atlantic Coastal Forest of Northeastern Brazil. Springer \& NYBG Press, New York, p. 193-220.

Gentry, A.H. 1974. Coevolutionary patterns in Central American Bignoniaceae. Annals of the Missouri Botanical Garden 61(3): 728-759.

Goulart, M.F.; Lemos Filho, J.P. \& Lovato, M.B. 2005. Phenological variation within and among populations of Plathymenia reticulata. Annals of Botany 96: 445-455.

Guedes, R.S.; Quirino, Z.G.M. \& Gonçalves, E.P. 2009. Fenologia reprodutiva e biologia da polinização de Canavalia brasiliensis Mart. ex Benth. (Fabaceae). Biotemas 22(1): 27-37.

Hernández-Conrique, D.; Ornelas, J.F.; García-Franco, J.G. \& Vargas, F. 2007. Nectar production of Calliandra longipedicellata (Fabaceae: Mimosoideae), an endemic Mexican shrub with multiple potential pollinators. Biotropica 39(4): 459-467.

Janzen, D.H. 1967. Synchronization of sexual reproduction of trees within the dry season in Central America. Evolution 21(3): 620637.

Juncá, F.A; Funch, L. \& Rocha, W. 2005. Biodiversidade e Conservação da Chapada Diamantina. Ministério do Meio Ambiente, Brasília.

Koptur, S. 1984. Breeding systems of Neotropical Inga trees. Evolution 38(5): 1130-1143.

Leyser, G.; Viniski, M.; Dinida, A.L.; Zanin, E.M. \& Budke, J.C. 2009. Espectro de dispersão em um fragmento de transição entre floresta ombrófila mista e floresta estacional na região do alto Uruguai, Rio Grande do Sul, Brasil. Pesquisas 60: 355366.

Lima, H.C.; Queiroz, L.P.; Morim, M.P.; Souza, V.C.; Dutra, V.F.; Bortoluzzi, R.L.C.; Iganci, J.R.V.; Fortunato, R.H.; Vaz, A.M.S.F.; Souza, E.R.; Filardi, F.L.R.; Valls, J.F.M.; Garcia, F.C.P.; Fernandes, J.M.; Martins-da-Silva, R.C.V.; Perez, A.P.F.; Mansano, V.F.; Miotto, S.T.S.; Tozzi, A.M.G.A.; Meireles, J.E.; Lima, L.C.P.; Oliveira, M.L.A.A.; Flores, A.S.; Torke, B.M.; Pinto, R.B.; Lewis, G.P.; Barros, M.J.F.; Schütz, R.; Pennington, T.; Klitgaard, B.B.; Rando, J.G.; Scalon, V.R.; Cardoso, D.B.O.S.; Costa, L.C.; Silva, M.J.; Moura, T.M.; Barros, L.A.V.; Silva, M.C.R.; Queiroz, R.T.; Sartori, A.L.B.; Camargo, R.A.; Lima, I.B.; Costa, J.; Soares, M.V.B.; Snak, C.; São-Mateus, W.; Falcão, M. J.; Martins, M.V.; Reis, I.P. \& Cordula, E. 2014. Fabaceae. In: Lista de Espécies da Flora do Brasil. Jardim Botânico do Rio de Janeiro. Disponível em http://floradobrasil.jbrj.gov.br/ jabot/floradobrasil/FB115; acesso em 10 jun. 2014. 
Liuth, H.S.; Talora, D.C. \& Amorim, A.M. 2013. Phenological synchrony and seasonality of understory Rubiaceae in the Atlantic Forest, Bahia, Brasil. Acta Botanica Brasilica 27: 195204.

Locatelli, E.M. \& Machado, I.C. 2004. Fenologia de espécies arbóreas de uma mata serrana (Brejo dos Cavalos) em Pernambuco, Nordeste do Brasil. In: K.C. Pôrto, J.J. Cabral \& M. Tabarelli (orgs), Brejos de Altitude: história natural, ecologia e conservação. MMA/PROBIO, Brasília, p. 255-276.

Marques, M.C.M.; Roper, J.J. \& Salvalaggio, A.P.B. 2004. Phenological patterns among plant life-forms in a subtropical forest in Southern Brazil. Plant Ecology 173(2): 203-213.

Matthews, M.L. \& Hopkinson, J.M. (1998). Evaluation of the pollination biology and fecundity of Calliandra calothyrsus at Walkamin, North Queensland, Australia. Queensland Department of Primary Industries and Department of Plant Sciences, Australia.

Milla, R.; Castro-Díez, P. \& Montserrat-Martí, G. 2010. Phenology of Mediterranean woody plants from NE Spain: Synchrony, seasonality, and relationships among phenophases. Flora 205: 190-199.

Miranda, L.A.P.; Vitória, A.P. \& Funch, L.S. 2011. Leaf phenology and water potential of five arboreal species in gallery and montane forests in the Chapada Diamantina; Bahia; Brazil. Environmental and Experimental Botany 70: 143-150.

Morellato, L.P.C. \& Leitão-Filho, H.F. 1990. Phenological strategies of tree species in a mesophytic forest on Japi Mountain, Jundiaí, São Paulo. Revista Brasileira de Biologia 50(1): 163-173.

Morellato, L.P.C.; Talora, D.C.; Takahasi, A.; Bencke, C.C.; Romera, E.C. \& Zipparro, V.B. 2000. Phenology of Atlantic rain forest trees: a comparative study. Biotropica 32(4b): 811 823.

Morellato, L.P.C.; Alberti, L.F. \& Hudson, I.L. 2010. Applications of circular statistics in plant phenology: a case studies approach. In: I.L. Hudson \& M. Keatley (eds), Phenological Research: methods for environmental and climate change analysis. Springer, Heidelberg, p. 357-371.

Navarro, T.; Oualidi, J.; Taleb, M.S.; Pascual, V. \& Cabezudo, B. 2009. Dispersal traits and dispersal patterns in an oroMediterranean thorn cushion plant formation of the eastern High Atlas, Morocco. Flora 204: 658-672.

Newstrom, L.E.; Frankie, G.W. \& Baker, H.G. 1994. A new classification for plant phenology based on flowering patterns in lowland tropical rain forest trees at La Selva, Costa Rica. Biotropica 26(2): 141-159.

Obermuller, E.A.; Nascimento, G.B.; Gava, H.Z.; Ribeiro, L.F. \& Silva, A.G. 2008. O contraste entre síndromes de polinização e sistemas efetivos de polinização e suas perspectivas para ecossistemas associados à Mata Atlântica. Natureza on line 6(1): 42-47.

Oliveira, M.I.B. \& Sigrist, M.R. 2008. Fenologia reprodutiva, polinização e reprodução de Dipteryx alata Vogel (Leguminosae-Papilionoideae) em Mato Grosso do Sul, Brasil. Revista Brasileira de Botânica 31(2): 195-207.

Ollerton, J.; Alarcón, R.; Waser, N.M.; Price, M.V.; Watts, S.; Cranmer, L.; Hingston, A.; Peter, C.I. \& Rotenberry, J. 2009. A global test of the pollination syndrome hypothesis. Annals of Botany 103:1471-1480.
Pedroni, F.; Sanchez, M. \& Santos, F.A.M. 2002. Fenologia da copaíba (Copaifera langsdoffii Desf. - Leguminosae, "Caesalpinioideae") em uma floresta semidecídua no Sudeste do Brasil. Revista Brasileira de Botânica 25(2): 183-194.

Pereira, T.S.; Costa, M.L.M.N.da; Moraes, L.F.D. \& Luchiari, C. 2008. Fenologia de espécies arbóreas em floresta atlântica da Reserva Biológica de Poço das Antas, Rio de Janeiro, Brasil. Iheringia 63(2): 329-339.

Pinto, A.M.; Morellato, L.P.C. \& Barbosa, A.P. 2008. Fenologia reprodutiva de Dipteryx odorata (Aubl.) Willd (Fabaceae) em duas áreas de floresta na Amazônia Central. Acta Amazônica 38(4): 643-650.

Primack, R.B. 1987. Relationships among flowers, fruits, and seeds. Annual Reviews of Ecology and Systematics 18: 409-430.

Quirino, Z.M.G \& Machado, I.C. 2014. Pollination syndromes in a Caatinga plant community in northeastern Brazil: seasonal availability of floral resources in different plant growth habits. Brazilian Journal of Biology 74(1): 62-71.

R Core Team. 2014. R: A Language and Environment for Statistical Computing. R Foundation for Statistical Computing, Vienna, Austria. Disponível em http://www.R-project.org/.

Ramírez, N. 2002. Reproductive phenology, life forms, and habitats of the Venezuelan Central Plain. American Journal of Botany 89: 836-842.

Reich, P.B. 1995. Phenology of tropical forests: patterns, causes and consequences. Canadian Journal of Botany 72(2): 164-174.

Ribeiro-Filho, A.A.; Funch, L.S. \& Rodal. M.J.N. 2009. Composição florística da floresta ciliar do rio Mandassaia, Parque Nacional da Chapada Diamantina, Bahia, Brasil. Rodriguésia 60: 265-276.

Rivera, J. \& Cozza, J. 2008. Reduced photoperiod induces partially synchronous flowering in an understory rain forest herb, Begonia urophylla in Costa Rica. Biotropica 40(3): 363365.

San Martin-Gajardo, I.S. \& Morellato, L.P.C. 2003. Fenologia de Rubiaceae do sub-bosque em floresta atlântica no sudeste do Brasil. Revista Brasileira de Botânica 26(3): 299-309.

Silva, A.L.G; Chaves, S.R \& Brito, J.M. 2011. Reproductive biology of Bowdichia virgilioides Kunth (Fabaceae). Acta Scientiarum 33(4): 463-470.

Souza, I.M.; Coutinho, K. \& Funch, L.S. 2012. Estratégias fenológicas de Senna cana (Nees \& Mart.) H.S. Irwin \& Barneby (Fabaceae: "Caesalpinioideae") como mecanismo eficiente para atração de polinizadores. Acta Botanica Brasilica 26(2): 435-443.

Staggemeier, V.G.; Diniz-Filho J.A.F. \& Morellato L.P.C. 2010. The shared influence of phylogeny and ecology on the reproductive patterns of Myrteae (Myrtaceae). Journal of Ecology 98: 1409-1421.

Stevenson, P.R.; Castellanos, M.C.; Cortés, A.I. \& Link, A. 2008. Flowering patterns in a seasonal tropical lowland forest in Western Amazonia. Biotropica 40(5): 559-567.

Talora, D.C. \& Morellato, P.C. 2000. Fenologia de espécies arbóreas em floresta de planície litorânea do Sudeste do Brasil. Revista Brasileira de Botânica 23(1): 13-26.

Tripp, E.A. \& Manos, P.S. 2008. Is floral specialization an evolutionary dead-end? Pollination system transitions in Ruellia (Acanthaceae). Evolution 62-7: 1712-1737.

Umaña, C.L.A. \& Alencar, J.C. 1993. Comportamento fenológico 
da sucupira-preta (Diplotropis purpurea (Rich.) Amsh. var. coriacea Amsh.) na Reserva Florestal Ducke. Acta Amazonica 23(1): 199-211.

Van der Pijl, L. 1982. Principles of Dispersal in Higher Plants. Springer-Verlag, New York.

Viana, B.F. \& Kleinert, A.M.P. 2006. Structure of bee-flower system in the coastal sand dune of Abaeté, Northeastern Brazil. Revista Brasileira de Entomologia 50(1): 53-63.
Williams-Linera, G. \& Meave, J. 2002. Patrones fenológicos. In: M.R. Guariquata \& G.H. Kattan (orgs), Ecologia $y$ Conservacion de Bosques Neotropicales. Universidad Nacional Autonoma, Libro Universitario Regional, Costa Rica, p. 407431.

Zalamea, M. \& González, G. 2008. Leaf fall phenology in a Subtropical Wet Forest in Puerto Rico: from species to community patterns. Biotropica 40(3): 295-304. 